\title{
Características deseables en un Laboratorio Remoto para la enseñanza de la física: indagando a los especialistas ${ }^{+*}$
}

\author{
Carlos Arguedas-Matarrita ${ }^{1}$ \\ Universidad Estatal a Distancia \\ San José - Costa Rica \\ Sonia Beatriz Concari ${ }^{1}$ \\ Universidad Nacional de Rosario \\ Rosario - Argentina
}

\section{Resumen}

El desarrollo de la tecnología asociada al acceso remoto en las últimas dos décadas ha posibilitado la creación y mejoramiento de numerosos laboratorios remotos para la enseñanza de la física, promoviendo su uso y posibilitando nuevos desarrollos. Sobre la base de la experiencia ya acumulada en el diseño, desarrollo y empleo de estos recursos, se plantea conocer las opiniones de los especialistas, que permitan identificar características deseables de los laboratorios remotos que orienten el desarrollo de nuevos laboratorios de acceso remoto. Se presentan resultados obtenidos de las opiniones fundadas de especialistas, relativos a las características tecnológicas y pedagógicas que deberían tener los laboratorios remotos para la enseñanza de la física. Los mismos serán útiles para el diseño de una propuesta de desarrollo de un laboratorio remoto en la Universidad Estatal a Distancia de Costa Rica.

Palavras-chave: Laboratorios Remotos; Experimentación; Educación a Distancia.

\footnotetext{
${ }^{+}$Desirable features in a Remote Lab for the Teaching of Physics: Interviewing the Experts

* Recebido: dezembro de 2017. Aceito: julho de 2018.

${ }^{1}$ E-mails: carguedas@uned.ac.cr; sconcari@gmail.com
} 


\begin{abstract}
The development of technology linked to remote access over the last two decades has allowed for the building and improvement of several remote labs for the teaching of physics, thus promoting their use and making new developments possible. On the basis of the experience already gained in design, development and use of these resources, it is necessary to know the opinions of experts which make it possible to identify the desirable features of remote labs that guide through the development of new remote access labs. The results shown are the ones obtained from the well-grounded views of experts, regarding the technological and educational features that remote labs for the teaching of physics should have. These will be useful for the design of a development proposal of a remote lab at Universidad Estatal a Distancia de Costa Rica.
\end{abstract}

Keywords: Remote Laboratorios; Experimentation; Distance Learning.

\title{
I. Introducción
}

En la actualidad las Tecnologías de la Información y la Comunicación (TIC) brindan una gran variedad de herramientas que, integradas a diversas actividades didácticas, facilitan el aprendizaje; una de estas tecnologías son los Laboratorios Remotos (LR), los cuales permiten la experimentación real utilizando una computadora con conexión a Internet, complementando el trabajo experimental de la física tanto en la modalidad presencial como en la modalidad a distancia. Por su naturaleza, los LR constituyen un complemento idóneo para la educación a distancia (EaD) (GARCÍA et al., 2016) ya que permiten el trabajo autónomo desde el lugar y en el momento que el estudiante lo disponga.

Si bien el LR está siendo empleado desde hace décadas en la educación superior, principalmente para la formación profesional de ingenieros, no ha sido una tecnología incorporada a programas de formación a distancia, o semipresenciales, tal como lo muestra una reciente revisión sistemática de la literatura (GONZÁLEZ; PERDOMO; PASCUAS, 2017).

El objetivo de este trabajo es mostrar parte de los resultados de una investigación doctoral que busca diseñar una propuesta para el diseño y desarrollo de un LR en la Universidad Estatal a Distancia de Costa Rica, resultados obtenidos referentes a las características que debería tener un LR para esta modalidad desde el punto de vista de una muestra de especialistas en el diseño, desarrollo y uso de estos recursos educativos. En trabajos previos se han identificado proyectos que ofrecen LR para la enseñanza de la física (ARGUEDAS; CONCARI, 2016a), así como sus características tecnológicas y pedagógicas (ARGUEDAS; CONCARI, 2016b) y se realizó un estudio para determinar las necesidades de 
un LR desde la óptica de los potenciales usuarios (ARGUEDAS-MATARRITA; CONCARI, 2017).

\section{I.1 Laboratorios Remotos}

Los LR son herramientas tecnológicas que integran software y hardware para configurar una experiencia real a la que se accede de manera remota a través de Internet o de redes académicas. El estudiante puede utilizar el LR para realizar actividades de laboratorio similares a las de un Laboratorio Tradicional (hands-on) (ZAMORA, 2012), con la diferencia de que las realiza a distancia. Las particularidades de los LR se detallan a continuación:

- Se trabaja con equipos reales.

- Incrementan el acceso a herramientas científicas, permitiendo a los estudiantes de todas las partes del mundo usarlas tanto a través de redes inalámbricas como celulares (móviles) (JOHNSON et al., 2013, p.16).

- Permiten una mayor utilización de los equipos de laboratorio, al estar disponibles 24 h, los 365 días del año (DA SILVA, 2006).

- Fomentan el trabajo autónomo, que es fundamental en el modelo actual de educación superior (DA SILVA, 2006).

- Permiten al profesor seguir los progresos de los estudiantes, a través de los Logs (historial) (SAN CRISTÓBAL, 2010, p.117).

- Proporcionan experiencias que los laboratorios tradicionales no pueden ofrecer, como el acceso a una gama mucho más amplia de equipos que son demasiado caros, peligrosos o logísticamente problemáticos (LABSHARE, 2017).

El uso de LR con carácter educativo se remonta a 1996; en la Universidad de Oregón, se desarrolla en ese año un laboratorio remoto de robótica para la enseñanza de la ingeniería (ATKAN, 1996) y en la Universidad de Tennessee, se lanza un plan piloto con seis experiencias remotas (HENRY, 1996). En 1997 se establece en Brasil el proyecto RExLab, dirigido a la enseñanza de la física tanto en el nivel medio como superior, siendo el proyecto pionero de experimentación remota de América del sur (DA SILVA et al, 2010); en 1998 se crea un LR de óptica en la Universidad de Stanford (HESSELINK et al., 2000), y le siguen el desarrollo de una plataforma de acceso remoto para un laboratorio de mecatrónica en la Universidad de Brooklyn (WONG; KAPILA; TZES, 2001), y un LR de microelectrónica en el Instituto Tecnológico de Massachusetts (DEL ALAMO et al., 2002).

En la actualidad, sólo de enseñanza de temas de física, se registran más de una decena de LR que ofrecen cerca de una centena de experimentos (ARGUEDAS, 2017).

El desarrollo de experiencias de acceso remoto hace posible que los estudiantes realicen prácticas de laboratorio a distancia, lo que los convierte en recursos idóneos para las instituciones de EaD, como es el caso de la UNED de Costa Rica. Al respecto, investigadores 
de la Universidad de Singapur anticipaban, hace ya veinte años, que esta forma de realizar experimentos sería un paso importante en la $\mathrm{EaD}$ (CHEN et al., 1999).

\section{I.2 Arquitectura de un LR}

Un LR permite trabajar sobre equipos reales por lo cual se requieren componentes de hardware y software que hagan posible la manipulación de dichos equipos. Para ello se diseña una arquitectura que haga posible este proceso, la arquitectura de un LR "es el "plan" con el que se conectan los dispositivos de hardware, software y los protocolos" (MARTín, 2013, p.19).

A continuación se describe brevemente la arquitectura general de un LR:

- Estudiante o usuario: accede por medio de una computadora o dispositivo móvil con conexión a Internet, si así lo permite el LR.

- Servidor web: encargado de mostrar el audio/video del laboratorio, las acciones que puede realizar sobre el laboratorio y los resultados de esas acciones (CASTRO et al., 2009).

- Servidor de base de datos: contiene la información del laboratorio (experimentos, datos de los alumnos, etc) (CASTRO et al., 2009).

- Laboratorio: equipo que se controla o manipula a distancia.

- Cámara web: muestra lo que está ocurriendo en el laboratorio.

En la Fig. 1 se muestra el esquema general de la arquitectura de un LR.

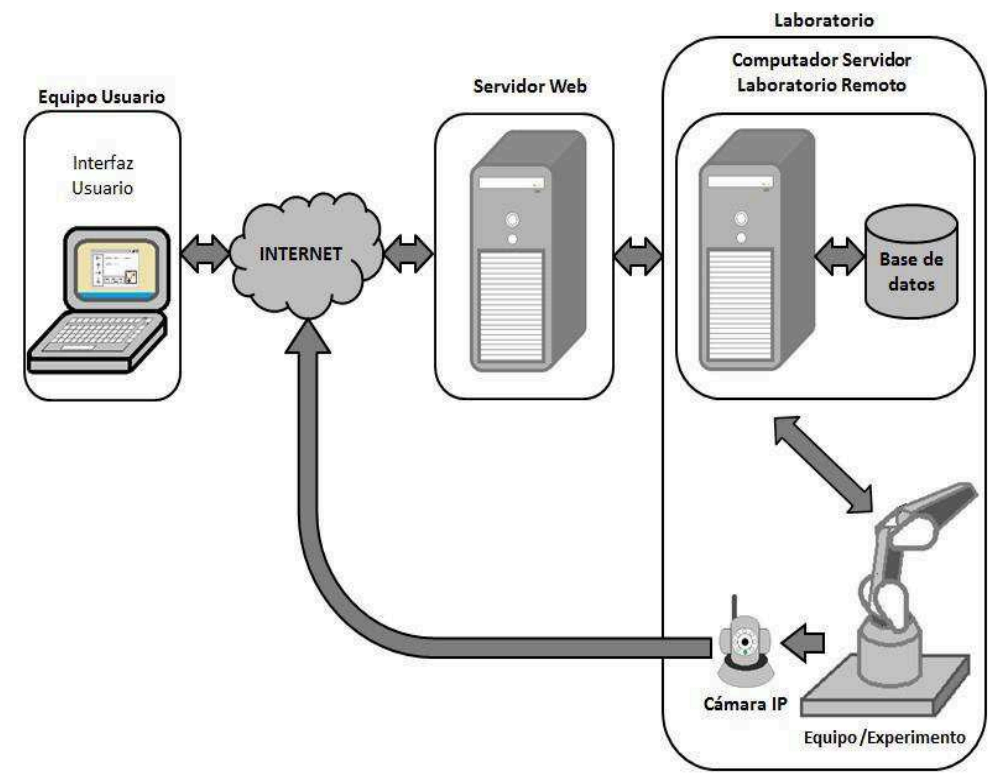

Fig. 1 - Arquitectura general de un LR (VELASQUEZ; RAMOS; AMAYA, 2016). 
Hay proyectos de LR que poseen arquitecturas más sencillas y en algunos casos no se cuenta con cámara web, sobre todo en las experiencias de circuitos eléctricos y electrónicos.

Existen otras arquitecturas más complejas que brindan recursos valiosos a usuarios, docentes e investigadores. Por ejemplo aquellas que permiten gestionar sus experiencias propias, además de otros LR. A los proyectos que presentan estas arquitecturas se les denomina RLMS (Remote Laboratory Management System).

\section{Metodología}

Se conformó una muestra intencionada integrada por especialistas de educación a distancia, física e ingeniería. Considerando como especialistas a aquellos profesionales vinculados a un proyecto de LR que posea prácticas de acceso remoto dirigidas a la enseñanza de la física.

Para la recolección de datos se confeccionó un cuestionario, el cual se sometió a un proceso de validación mediante el juicio de expertos, definido por ESCOBAR-PÉREZ y CUERVO-MARTÍNEZ (2008) "como una opinión informada de personas con trayectoria en el tema, que son reconocidas por otros como expertos cualificados en éste, y que pueden dar información, evidencia, juicios y valoraciones" (p.29). Para la selección de los expertos que validarían el cuestionario se estableció que debían cumplir con alguno(s) de los siguientes criterios:

- Estar vinculados a la investigación educativa.

- Ser especialistas en la enseñanza de la física y/o ingeniería.

- Ser especialistas en didáctica de las ciencias.

- Estar vinculados al desarrollo o uso de un LR.

En este proceso se contó con la participación de once expertos (se envió a un total 18 expertos) provenientes de los países: Argentina, Brasil, Costa Rica, Cuba, España y México. Atendiendo a las observaciones realizadas por los expertos, se eliminaron preguntas, se reformularon otras y se agregaron nuevas preguntas, resultando un instrumento de 18 preguntas; agrupadas en tres partes: datos generales, aspectos tecnológicos y aspectos pedagógicos relativos a los LR. El cuestionario se tradujo al idioma inglés ya que muchos de los especialistas a ser encuestados se encuentran en países donde no se habla español.

La versión definitiva del cuestionario se alojó en la herramienta Formularios de Google Drive, por lo que se aplicaron de forma auto-administrada. La dirección web del cuestionario fue enviada por correo electrónico de forma personalizada a especialistas que participan en proyectos de LR. Además se contó con la colaboración del coordinador del proyecto VISIR+ Plus promovido por la Unión Europea, quien envió un correo electrónico a 
sus contactos personales invitándolos a participar en el estudio. La recolección de información se realizó durante los meses de junio y julio.

Una vez que se recolectó la información, se revisaron las bases de datos que confeccionó la herramienta Formularios de Google Drive. Además se procedió a unificar los datos ya que, como ya se mencionó, el cuestionario se aplicó tanto en español como en inglés; el análisis estadístico se realizó utilizando el programa SPSS-17 (Statistical Package for the Social Sciences).

\section{Resultados y análisis}

Se contó con la participación de un total 40 especialistas, de los cuales 23 contestaron el cuestionario en idioma inglés y 17 el correspondiente en idioma español.

\section{III.1 Datos generales}

La edad promedio de la muestra fue de 42 años con una desviación estándar de 12 años. Con respecto al grado académico de los especialistas, el 60\% $(n=24)$ corresponde al grado de Doctor, el 25\% ( $\mathrm{n}=10)$ a Magister, el 10\% $(\mathrm{n}=4)$ son Licenciados y por último se registró un técnico $(2,5 \%)$ y un estudiante $(2,5 \%)$. Es importante resaltar que todos los especialistas en LR consultados pertenecen a instituciones universitarias, y que el $95 \%$ de los mismos son académicos con experiencia y reconocimiento de sus pares. La muestra proviene de 17 países diferentes como se muestra en la Fig. 2.

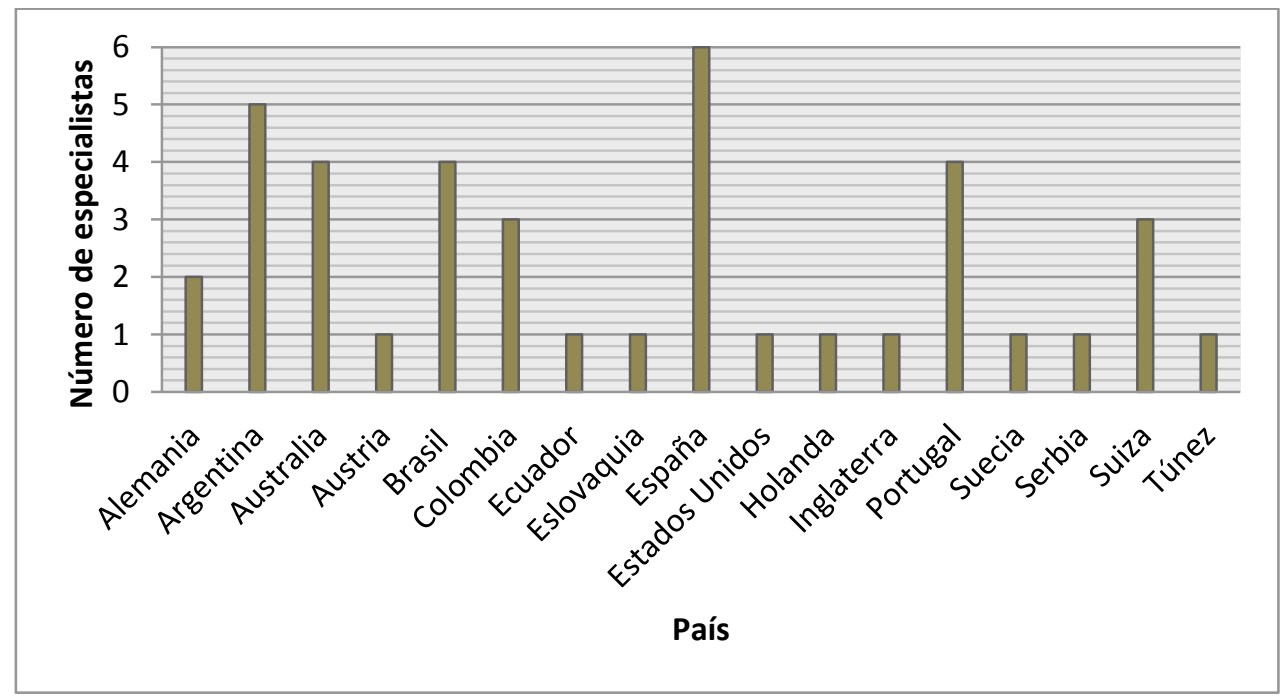

Fig. 2 - Países de procedencia de los especialistas.

En relación a la distribución por país, hay una mayor participación de especialistas de España $(n=6)$, los cuales se encuentran asociados a los proyectos: Web-Deusto, LabsLand, UNED-Labs, VISIR, Legos, Automática LabVIEW y Control y Física de la 
UNED. Seguido de Argentina $(n=5)$, los cuales trabajan en los proyectos: Laboratorio Remoto Grupo Galileo, Laboratorio remoto de Física Electrónica y VISIR, Calefones Solar FCEIA-UNR y el Laboratorio Remoto de Óptica-Ondas-Resonancia de la UBA. Con una frecuencia de cinco participantes, se encuentran los países Australia, Brasil y Portugal, de proyectos UTS RemoteLabs, USQ Labs, iLabs-Radioactivity; RexLab, RELLE - Remote Labs Learning Environment, Robótica; Physicslabfarm-VISIR y el e-lab.tecnico.ulisboa.pt. De Colombia y Suiza se tuvo la participación de tres especialistas de cada país que forman parte de los proyectos: Physilab, Laboratorio remoto para la enseñanza de la física; Labsfor control education. De Alemania participaron dos especialistas de los proyectos: Control Engineering y del Spectrometer@VirtualRemoteLab.net.

Por último completó el instrumento un solo participante de los siguientes países: Austria (VISIR, Radioactivitylab, black-bodyradiation); Ecuador (Laboratorio Remoto de Física); Estados Unidos (Remote Laboratory at University of Houston, SMSL); Holanda (GoLab); Inglaterra (Integration of virtual worlds/games and Remote Labs); Suecia (VISIR); Serbia (NERELA); Slovaquia (RL's for automatic control) y Túnez (Heat Transfer RL).

\section{III.2 Aspectos tecnológicos}

Con el fin de determinar el lenguaje de programación con el que se ha diseñado la aplicación web del LR en el que participa cada especialista, se planteó el interrogante: ¿En qué lenguaje de programación se ha elaborado la aplicación web de su Laboratorio Remoto?, en la Fig. 3 se muestran los resultados obtenidos.

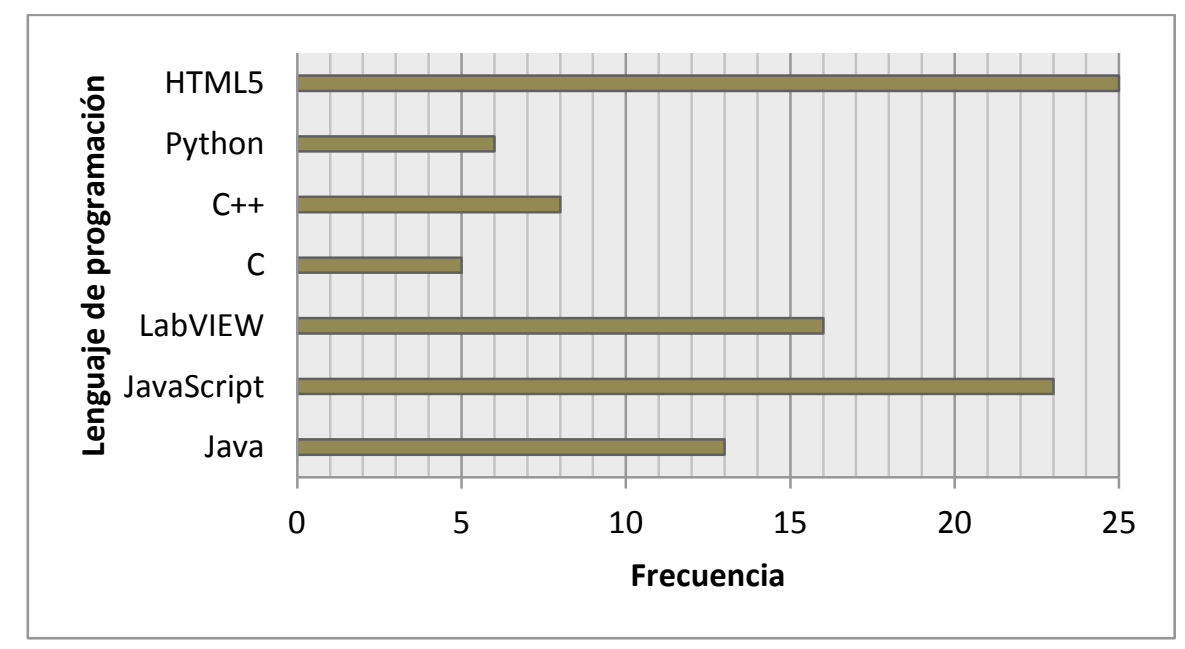

Fig. 3 - Lenguaje en el que se ha diseñado la aplicación web del LR.

Con respecto al lenguaje de programación utilizado, hay preferencia por lenguajes HTML5, JavaScript y LabVIEW. Estos lenguajes permiten el acceso desde diferentes dispositivos. Con respecto a las ventajas que brinda el lenguaje utilizado, los especialistas señalan las siguientes: 
- Interfaces amigables (LabVIEW, C++).

- Compatibilidad con hardware (LabVIEW).

- Una interface gráfica muy buena y alta intuitividad (Python, PHP ASP).

- El lenguaje C permite acceso al hardware, JavaScript facilidad en la presentación de datos.

- Facilidad ya que el código en HTML5 permite visualizar en cualquier dispositivo y no es dependiente ni de sistema operativo ni de aplicación.

- Java es inherentemente amigable para la web e integra bien con muchas otras plataformas y aplicaciones.

- Acceso a datos vía web y control de equipos externos con una computadora de escritorio (Java, Delphi).

- LabVIEW: acceso directo al hardware, muy eficiente.

- Funciona en todos los navegadores, sin necesidad de instalar y complementos (JavaScript, HTML5).

- LabVIEW está predefinido para el equipo de NI que usamos principalmente.

- Multiplataforma (JavaScript, C, HTML5).

- Cada uno es distinto, depende de la aplicación y el entorno. HTML5 es el mejor y más flexible actualmente aunque LabVIEW es más rápido.

- HTML5 y JavaScript se ejecutan en dispositivos móviles. PHP se utiliza en el lado del servidor para gestionar los procesos de autenticación. El hardware está controlado por un servicio de Windows programado en $C$ \# para mayor confiabilidad y escalabilidad (la biblioteca .net se puede usar en otros proyectos).

- No requiere plugins de navegador adicionales para ser usados (JavaScript, HTML5).

Se puede inferir que la elección del lenguaje de programación utilizado en la aplicación web del LR se define por la facilidad de acceso, además de que los LR puedan ser utilizados en distintas plataformas o dispositivos, y por último por la compatibilidad con el Hardware.

Para indagar sobre la colaboración y gestión del proyecto de LR en el que participan los especialistas, se formularon las preguntas: ¿Comparte laboratorios con otras instituciones?

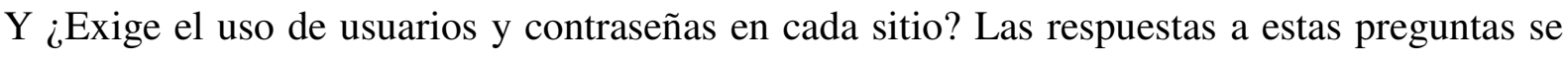
presentan en la Fig. 4.

Según las respuestas, un $82,2 \%(\mathrm{n}=33)$ indica compartir sus LR con otras instituciones, de ellos, el 62,2\% $(\mathrm{n}=25)$ indica no solicitar usuarios y contraseñas en sus prácticas de acceso remoto. Este aspecto es muy alentador ya que permite que instituciones que no cuentan con LR puedan disponer de prácticas de los proyectos que comparten sus LR 
y aún más, de aquellos que no solicitan datos de autenticación, lo que facilita el uso de estos recursos.

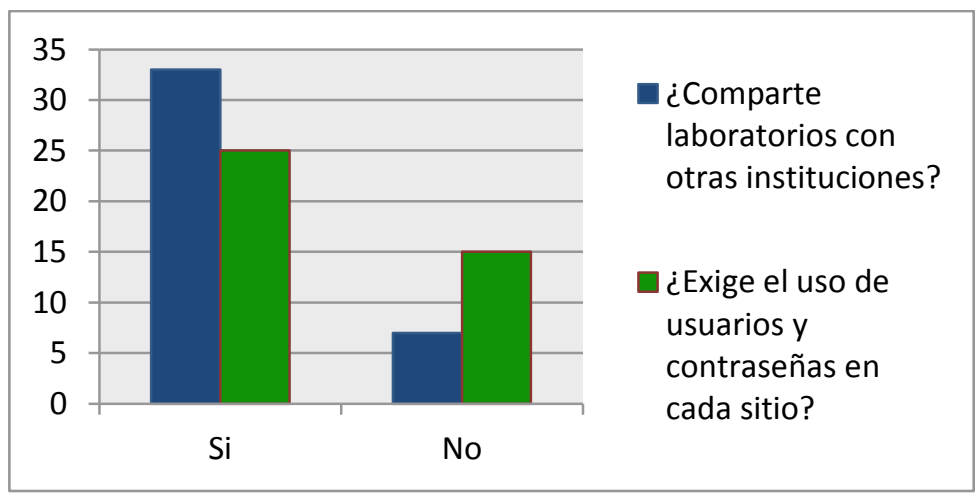

Fig. 4 - Respuestas a las preguntas ¿Comparte laboratorios con otras instituciones?

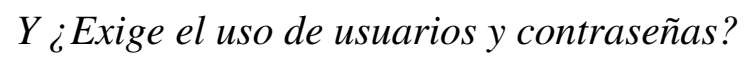

Debido a que en la EaD la utilización de plataformas educativas se ha vuelto muy habitual, se planteó la pregunta: ¿Considera conveniente gestionar un LR a través de un LMS (Learning Management System)?

Se obtuvo que un 75,0\% ( $\mathrm{n}=30)$ señala que es conveniente realizar la gestión del LR con un LMS, mientras que el 25,0\% $(\mathrm{n}=10)$ indicó que no es conveniente. Con el fin de determinar la relación entre la percepción de la conveniencia de gestionar el LR, con los años de trabajar en un proyecto de esta naturaleza, se analizaron las respuestas dividiendo la muestra participante en dos grupos: los que tienen más de 10 años de estar trabajando en el proyecto y los especialistas con menos de 10 años, los resultados obtenidos se muestran en la Tabla 1.

Hay asociación inversa estadísticamente significativa $(p=0,015)$ entre los años de trabajar en el proyecto de LR y el hecho de considerar conveniente gestionar el LR por medio de un LMS. Los especialistas que tienen más de diez años asociados a estos proyectos consideran que no es conveniente gestionar el LR con un LMS por las siguientes razones:

- LMS puede ser un factor limitante si alguien quiere probar el LR.

- Los LMS tienden a dejar de ser funcionales. Los estudiantes usan otras plataformas, principalmente las redes sociales.

- Optamos por que las prácticas sean de libre acceso y los LMS requieren que los usuarios se registren; usamos el LMS sólo para facilitar apoyos didácticos.

- No es necesario, se vuelve tedioso para el usuario.

- Debe ser una decisión del docente que utilizará el LR. 
Tabla 1 - Conveniencia de gestionar el LR con un LMS según los años de trabajar en proyectos de LR.

\begin{tabular}{|c|c|c|c|c|}
\hline \multirow{2}{*}{\multicolumn{2}{|c|}{ Años de trabajar en el LR }} & \multicolumn{2}{|c|}{$\begin{array}{l}\text { ¿Considera conveniente } \\
\text { gestionar un LR a través de un } \\
\text { LMS? }\end{array}$} & \multirow{3}{*}{$\frac{\text { Total }}{32}$} \\
\hline & & No & Sí & \\
\hline \multirow[t]{2}{*}{ Menos de10 } & Recuento & 5 & 27 & \\
\hline & Porcentaje & $15,6 \%$ & $84,4 \%$ & $100,0 \%$ \\
\hline \multirow[t]{2}{*}{ Más de 10} & Recuento & 5 & 3 & 8 \\
\hline & Porcentaje & $62,5 \%$ & $37,5 \%$ & $100,0 \%$ \\
\hline \multirow{2}{*}{\multicolumn{2}{|c|}{ Total }} & 10 & 30 & 40 \\
\hline & & $25,0 \%$ & $75,0 \%$ & $100,0 \%$ \\
\hline
\end{tabular}

Los especialistas con más experiencia ven como un factor negativo gestionar el LR con un LMS ya que el registro que exige una plataforma educativa puede limitar el uso del LR y, por ejemplo, un usuario que no se encuentre matriculado en un determinado curso no podría utilizar un LR que le parezca funcional para sus intereses educativos. Por ello, estos especialistas han permitido el uso libre de sus LR, lo cual es un factor muy positivo ya que el recurso puede ser utilizado sin la necesidad de solicitar permisos a las instituciones donde se encuentra el LR.

Cabe resaltar que los especialistas que tienen más de diez años están asociados a los proyectos: WebLab-Deusto, RexLab, Go-Lab y VISIR, los que han sido pioneros en compartir sus experiencias con otras instituciones, fomentando el trabajo experimental con estos recursos tanto en la escuela media como universitaria.

Los especialistas que tienen menos de diez años de trabajar en un proyecto de LR que consideran que sí es conveniente gestionar el LR con LMS, señalan razones como las que se mencionan:

- Integración en una única plataforma.

- Si implica poder gestionar la configuración específica de un laboratorio (p.ej. por un profesor) podría en efecto ser conveniente hacerlo en el LMS que es donde quizás lo despliegue. Si se refiere a configuración interna del RLMS, sin embargo, tendría menos sentido.

- La integración del laboratorio con la parte educativa de las asignaturas o tareas es fundamental como complemento educativo.

- Puesto que ayudaría a la formación complementaria fuera de las aulas.

- Permite organizar mejor la administración de los usuarios. 
- Para integrarlo como un área de pruebas (playground) para estudiantes de física.

- De esta manera es transparente el acceso al laboratorio y el acceso es únicamente a través de la plataforma virtual.

- Facilidad de acceso y complementación con otros recursos.

- Da facilidad en la gestión de usuarios.

- Se utiliza el LR en MOODLE, brinda facilidad al estudiante.

- Hay grupos de trabajo que han tenido buenos resultados.

- Para el uso del experimento en el entorno de aprendizaje, esto brinda un mejor escenario educativo.

Es claro que estas posiciones refieren a tanto a cuestiones de gestión y administración de usuarios, por parte de los administradores del LR, como a aspectos vinculados con las facilidades que el estudiante podría tener desde un marco institucional. Sobre esta última cuestión, las opiniones positivas referidas, en su gran mayoría, a la integración de los LR en una única plataforma, reflejan lo que en programas de EaD, podría facilitar el aprendizaje de los estudiantes, al tener en un mismo espacio todos los recursos necesarios para el desarrollo de las distintas actividades del curso.

Por otra parte se consultó mediante una Grilla sobre el grado en el que se han tenido en cuenta algunos aspectos al diseñar el LR en el que trabaja el especialista consultado. Los resultados se presentan en la Fig. 5.

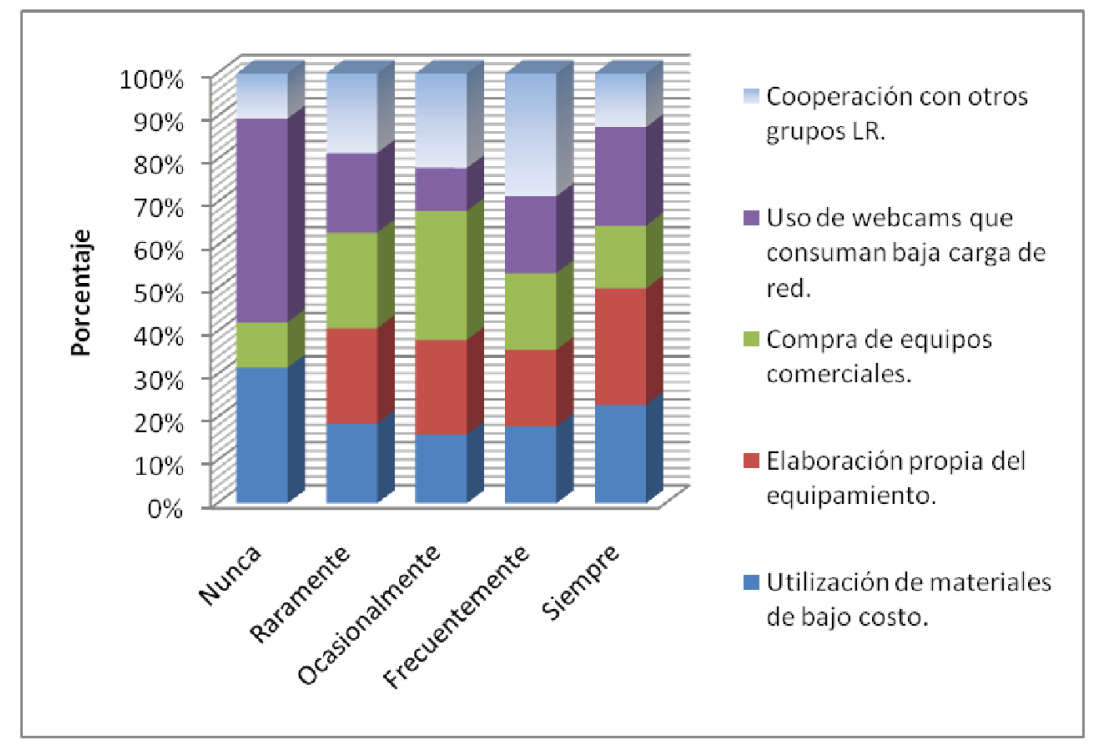

Fig. 5 -Aspectos que se han tenido en cuenta por parte de los especialistas al diseñar su LR.

Según estos especialistas, en el diseño de los LR del proyecto con el que están vinculados, la elaboración propia del equipamiento es un aspecto común sine qua non, siendo la utilización de materiales de bajo costo y la cooperación con otros grupos de trabajo, 
cuestiones que los especialistas mencionan también haber tenido en cuenta en el diseño de sus LR. Sin embargo hay un porcentaje considerable para la opción compra de equipos comerciales, un aspecto que se puede tomar en cuenta en proyectos que están iniciando ya que la compra de un LR puede ser el punto de partida para el establecimiento y crecimiento de un proyecto de esta naturaleza.

Por otra parte, de la Fig. 5 se puede ver que a pesar de que el uso de cámaras web, que consuman baja carga de red, es un aspecto importante para el uso del LR, sobre todo en zonas donde la conexión no es muy buena, es un aspecto que una gran parte de los participantes no ha tomado en cuenta en el diseño de sus LR.

\section{III.3 Aspectos pedagógicos}

Cuando se utiliza un LR como recurso educativo se requieren materiales didácticos y de apoyo para el estudiante que permitan una fácil y correcta utilización de los mismos, por ello se indagó sobre los apoyos educativos que ofrecen las experiencias de acceso remoto de los especialistas. Los resultados se muestran en la Fig. 6.

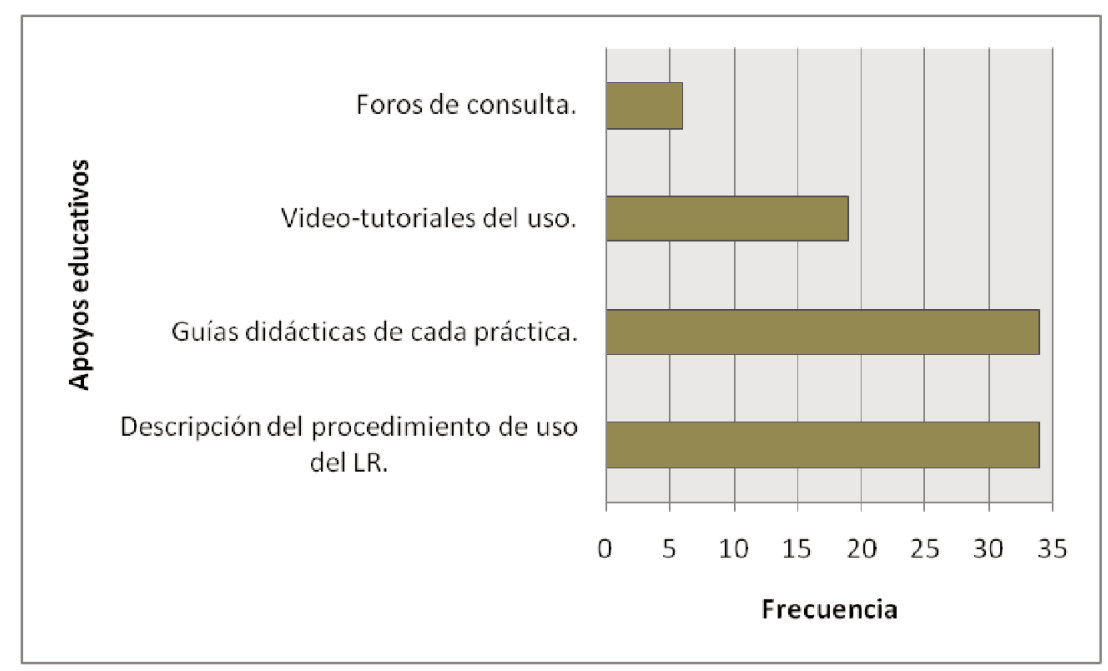

Fig. 6 - Apoyos educativos de los LR.

En su gran mayoría ( $\mathrm{f}=34$ ) los recursos de apoyo educativo más utilizados por los especialistas son las guías didácticas de cada práctica y la descripción del procedimiento de uso del LR, seguido de los video-tutoriales $(f=19)$ y por último los foros de consulta $(f=6)$. Como otros apoyos educativos se mencionaron además los siguientes:

- Cuando se utilizan LR en MOOC (Massive Open Online Courses) se emplea el entorno MOODLE como complemento y repositorio.

- El portal del proyecto cuenta con materiales de apoyo técnico y educativo. 
- Se realiza una presentación en las clases presenciales por el profesor con el fin de que conozcan el entorno en el que deben realizar las prácticas.

- Al ser un proyecto nuevo los recursos de apoyo educativo están en proceso de elaboración.

Los proyectos de LR que se han desarrollado a nivel mundial y de los cuales los especialistas participantes de este estudio son miembros, pertenecen a universidades de modalidad presencial que han buscado apoyar el trabajo experimental con estos recursos; por esta razón, muchas de las explicaciones referidas al empleo educativo de los LR se ofrecen en las sesiones presenciales.

Como respuesta a la consulta sobre si consideraban que los recursos de apoyo educativo de su proyecto eran suficientes y adecuados, un 50,0\% $(\mathrm{n}=20)$ de la muestra señaló que sí, mientras que el restante 50,0\% indicó lo contrario.

Algunos comentarios de los especialistas que consideran que los apoyos educativos de sus proyectos no son suficientes ni adecuados son:

- Falta de financiación.

- Poco apoyo en las instituciones.

- Faltan algunos desarrollos de tutoriales.

- Falta mayor colaboración con el personal académico.

- Nunca es suficiente cuando se trata de apoyos educativos.

- Falta personal para mejorar aspectos educativos para el uso del LR.

Según estas opiniones, la falta de mejores apoyos educativos para el uso de los LR se debe a razones de financiamiento que limita el personal necesario para trabajar en el desarrollo de los mismos. Por otra parte, los especialistas que indican que los apoyos educativos son los adecuados, expresan aspectos como los siguientes:

- Según el feedback recibido por nuestros alumnos, sí, considero que son suficientes y adecuados.

- No hemos tenido inconvenientes en el uso de los experimentos.

- Así lo manifiestan los alumnos y el resultado del uso de laboratorio.

- Teniendo en cuenta la capacidad de los usuarios y la facilidad de manejo.

- Con la experiencia que se tiene en el proyecto de ocho años los apoyos se adecuan a las nuevas demandas de los usuarios.

- Utilizamos interfaces de usuario ajustables como andamios implícitos para los estudiantes.

Estas opiniones positivas se basan en las experiencias y percepciones de los estudiantes, los cuales son un buen referente ya que son quienes realmente le dan valor al LR, 
como usuarios directos, una vez desarrollados, y quienes los utilizan para fortalecer su aprendizaje.

Acerca de las ventajas educativas que poseen los LR en la EaD desde la visión de los especialistas, se obtuvieron los resultados que se muestran en la Fig. 7.

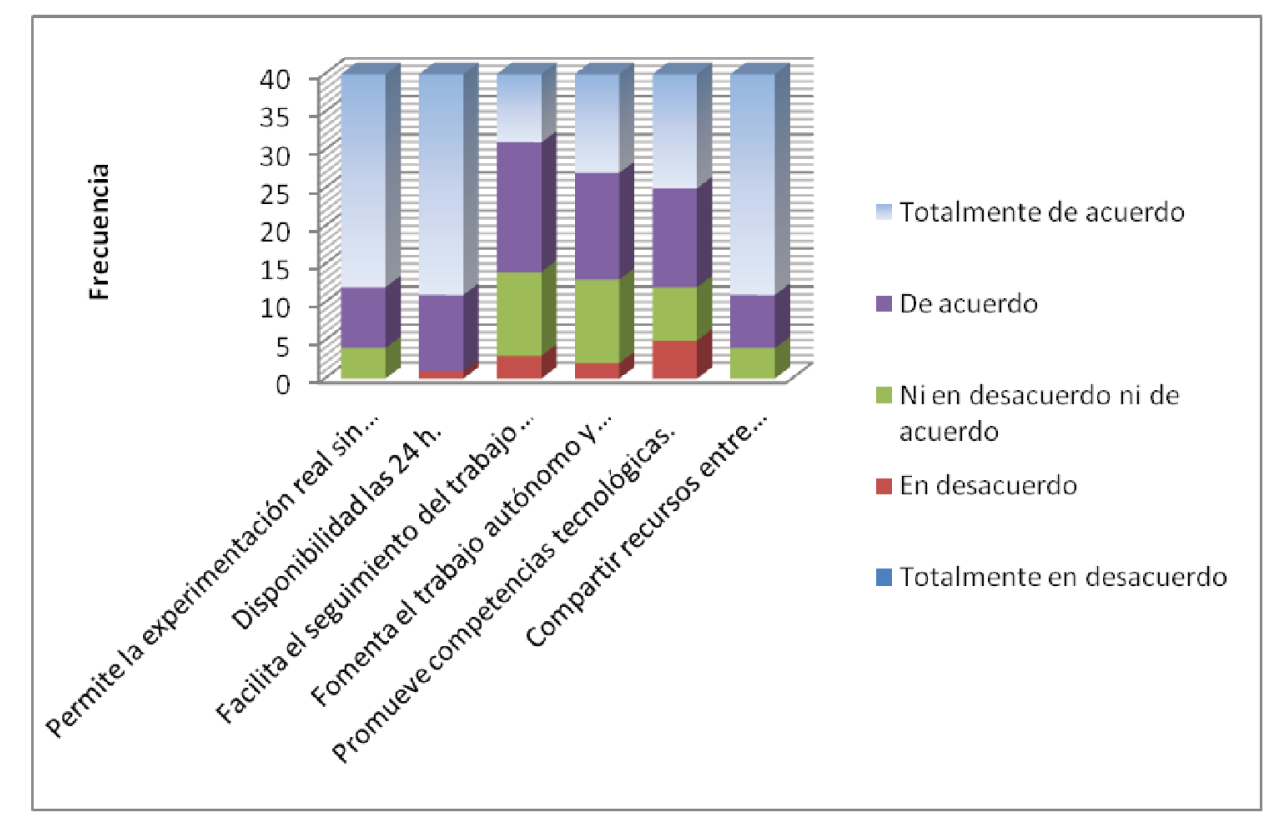

Fig.7 - Ventajas que ofrecen los LR en la EaD.

Un $90 \%$ de los participantes indica estar entre Totalmente de acuerdo y De acuerdo en que los LR: permiten la experimentación real sin tener que desplazarse, están disponibles las $24 \mathrm{~h}$ y permiten que se compartan recursos entre instituciones.

Se consultó también sobre las tres características principales que, como especialistas, consideran que se deben tener en cuenta al diseñar un LR; algunas de las respuestas se muestran a continuación:

- Alineado con los objetivos de una asignatura; fácil de usar; con resultados de aprendizaje claros.

- Facilidad, auto-explicativo y dinámico.

- Integración con la materia, definición clara de objetivos y competencias a alcanzar, uso de recursos educativos para la resolución.

- Realizar las especificaciones conjuntamente con los docentes que podrán utilizarlos; integrarlos a los planes de clases/estudios y no sólo ponerlos a disposición; que puedan dar retroalimentación a los alumnos.

- Experimentación de los fenómenos físicos, inclusión de instituciones que no tienen laboratorios y fusión de los contenidos teóricos de la asignatura con las prácticas.

- Interactividad, simplicidad e ilustración visual. 
- Debe ser fácil de usar, intuitivo, brindar datos reales del experimento y facilitar la compresión del tema al alumno.

- Experimentación sobre dispositivos no accesibles localmente, atemporalidad, consideración de los procesos cognitivos del estudiante.

- Facilidad de uso. Similitud con los dispositivos reales. Intuitivo.

- Repetibilidad, credibilidad (ayudar al usuario a entender qué es un dispositivo físico no una simulación y cualquier desviación de los resultados esperados no son fallas del sistema), confiabilidad.

- Simplicidad, pequeños experimentos que se pueden completar en pocos minutos, un laboratorio remoto debe ser pedagógicamente agnóstico (es decir, puede ser utilizado por diferentes profesores para diferentes fines).

- No restringir el tiempo o ubicación, que se pueda rehacer el laboratorio muchas veces.

- El LR debe proporcionar una experiencia clara e intuitiva (los estudiantes deben saber lo que están haciendo), atractivo (el experimento debe dar sentido y relación con problemas reales), utilidad educativa (los estudiantes deben ser capaces de llevar a cabo las tareas asignadas), y por supuesto el LR debe alentar a los estudiantes a "tratar de aprender".

- Los desarrolladores deben obtener los requisitos docentes, el material de aprendizaje debe proporcionarse, la interfaz de usuario de la aplicación debe ser fácil de usar.

- Simplicidad de uso, autonomía total y claridad de los objetivos educativos.

- Equipo robusto, objetivos educativos, facilidad de uso.

- Fácil de usar (la mayoría de los estudiantes son principiantes con las tecnologías); experiencia con la manera en que muchos científicos profesionales hacen ahora la investigación; equidad - cualquier escuela de cualquier lugar tiene acceso a la misma tecnología y datos.

- Accesibilidad, facilidad de uso, escalabilidad, sostenibilidad (Conformación de un equipo de trabajo).

Una de las características mencionadas con mayor frecuencia por los especialistas, es que el LR sea de fácil acceso. Éste, sin duda, es un aspecto muy importante en el desarrollo de estos recursos. Además, como señaló uno de los especialistas, el diseño de un LR se debe pensar en que pueda ser útil para diferentes usuarios ya sea de la escuela media o para la formación superior.

Por último se consultó sobre el tiempo en que el LR, en el que el especialista está involucrado, estuvo inactivo el último año, obteniéndose los resultados que se muestran la Fig. 8. 
Pese a que una de las características esperables de un LR es su disponibilidad las 24 horas, todos los días del año, casi la mitad $(47,5 \%)$ indicó que su proyecto no estuvo disponible un máximo del $10 \%$ del año. Un 15,0\% de los especialistas reveló que su LR no estuvo activo entre el 11 y $20 \%$ del tiempo, lo que representa que un 62,5\% de los proyectos en los que trabajan los especialistas estuvieron disponibles un $80,0 \%$ del tiempo en el último año. La no disponibilidad del $100 \%$ del tiempo estaría dada por la necesidad de mantenimiento y/o reparación de los equipos reales que integran los LR, los que son susceptibles de sufrir desperfectos mecánicos o eléctricos debido al uso, así como a necesidades de actualizaciones del software.

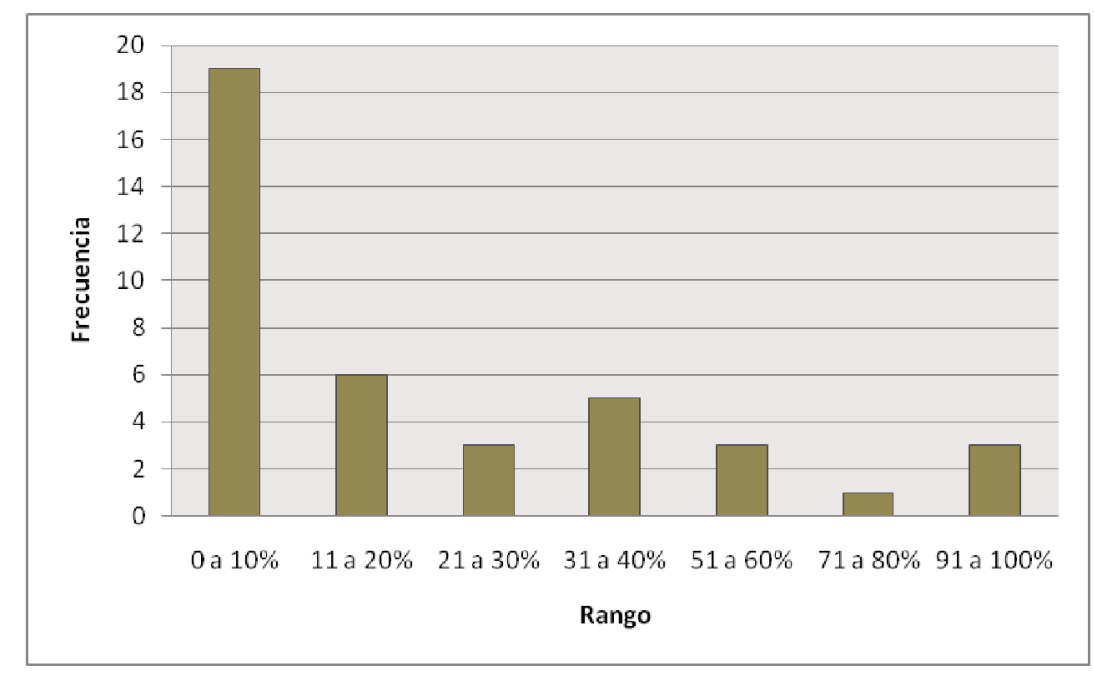

Fig. 8 - Frecuencia de respuestas sobre el porcentaje de tiempo en el último año, en el que el LR no estuvo disponible.

\section{Conclusiones}

En esta investigación se logró contar con la participación de especialistas de los proyectos de LR de diversas partes del mundo, los cuales coinciden en que en el desarrollo de estos recursos se busca que se pueda acceder a ellos desde distintos dispositivos, es decir que sean multiplataforma. Esto puede mediante lenguajes de programación que lo permiten como HTML5 y JavaScript entre otros, lo que facilita el acceso y mayor uso de estos recursos educativos.

Según los especialistas hay una tendencia en compartir sus experimentos con otras instituciones, fomentando el trabajo colaborativo de investigadores y docentes, y promoviendo la experimentación en los estudiantes, lo que permite ampliar las posibilidades de realizar experimentos reales en las instituciones, regiones o países que no cuentan con estos recursos educativos.

La gestión del LR con una plataforma educativa es bien vista por los especialistas ya que permite integrar los recursos educativos en un mismo entorno, facilitando los apoyos 
requeridos para el uso efectivo del LR. Sin embargo también se ve esta integración como un problema sobre todo para el uso libre del LR ya que para ingresar a una plataforma educativa (LMS) se requiere completar un proceso de registro.

De acuerdo con las consideraciones de los especialistas, la falta de mejores apoyos educativos para el uso de los LR se debe a razones de financiamiento. Desde esta óptica, es importante que un proyecto de desarrollo de estos recursos educativos tenga el apoyo institucional que provea el financiamiento suficiente, que permita el crecimiento, mejoramiento y fortalecimiento del mismo.

El desarrollo que ha tenido en las últimas dos décadas la tecnología asociada al acceso remoto ha posibilitado la creación y mejoramiento de numerosos LR para la enseñanza de la física, mayoritariamente destinados a la educación universitaria de carreras científico tecnológicas que tienen a la física como asignatura de formación básica y/o específica.

Conocer las opiniones de los especialistas, basadas en la experiencia de los mismos en el diseño, desarrollo y empleo de LR, constituye un aporte valioso para el desarrollo de nuevos LR.

\section{Agradecimientos}

El primer autor agradece a la Universidad Estatal a Distancia (UNED) por la beca otorgada a través del Acuerdo de Mejoramiento Institucional (AMI) para la realización del Doctorado en Educación en Ciencias Experimentales en la Universidad Nacional del Litoral, Argentina.

Este trabajo ha sido realizado en el marco del proyecto 1ING505: "Procesos educativos mediados por tecnologías en ciencias e ingeniería. Estudio de casos" de la Universidad Nacional de Rosario (UNR).

\section{Referencias}

ARGUEDAS, C. Diseño y desarrollo de un Laboratorio Remoto para la enseñanza de la física en la UNED de Costa Rica. 2017. Tesis (Doctorado en Educación en Ciencias Experimentales) - Universidade Nacional do Litoral, Santa Fe. Disponible en: <http://bibliotecavirtual.unl.edu.ar:8080/tesis/handle/11185/1018>. Acceso en: 2 mar. 2018.

ARGUEDAS-MATARRITA, C.; CONCARI, S. B. Desirable Features in a Physics Remote Laboratory: users' opinions. International Journal of Advancement in Education and Social Sciences, v. 5, n. 2, 1-7, 2017. Disponible en: <http://www.irosss.org/ojs/index.php/ IJAESS/article/view/857>. Acceso en: 16 ago. 2018.

ARGUEDAS, C.; CONCARI, S. B. Remote laboratories used in physics teaching: a estate of the art. En: INTERNATIONAL CONFERENCE ON REMOTE ENGINEERING AND VIRTUAL INSTRUMENTATION, $13^{\text {th }}, 2016$ a, Madrid, España. 
ARGUEDAS, C.; CONCARI, S. B. Laboratorios remotos para la enseñanza de la física: características tecnológicas y pedagógicas, Revista de Enseñanza de la Física, v. 28, n. Extra, 235-243, 2016b. Disponible en: <https://revistas.unc.edu.ar/index.php/revistaEF /article/ view/15644/15451>. Acceso en: 05 dez. 2017.

ATKAN, B. Distance Learning Applied to Control Engineering Education. 1996. Tesis (Master of Science in Electrical and Computer Engineering). Disponible en: $<$ https://ir.library.oregonstate.edu/xmlui/bitstream/handle/1957/34806/AktanBurcin1996.pdf? sequence=3>. Acceso en: 18 ago. 2018.

CASTRO, M. et al. Servicios para Plataformas Educativas: Laboratorios y Aplicaciones. En: CONGRESO DE TECNOLOGÍAS APLICADAS A LA ENSEÑANZA DE LA ELECTRÓNICA, IX, 2009, Madrid, España.

CHEN, S. et al. Development of Remote Laboratory Experimentation through Internet. En: PROCEEDINGS OF THE 1999 IEEE HONG KONG SYMPOSIUM ON ROBOTICS AND CONTROL, 1999, Hong Kong. Disponible en: <ftp://labattmot.ele.ita.br/ele/lfilipe/Lab _Real_Remoto/Artigos/Lab_Remoto/Chen_SH_Dev_Remote_Lab_Experimentation_Throug h_Internet.pdf>. Acceso en: 14 jun. 2015.

DA SILVA, J. B. et al. Experimentação Remota em Santa Catarina. Revista Técnico Científica do IFSC, v. 1, n. 1, 84-87, 2010.

DA SILVA, J. B. A utilização da experimentação remota como suporte para ambientes colaborativos de aprendizagem. 2006. Disertación (Doctoral) - Universidad Federal de Santa Catarina.

DEL ALAMO, J. et al. The MIT Microelectronics WebLab: a Web-Enabled Remote Laboratory for Microelectronic Device Characterization, 2002. Disponible en: <http://www-mtl.mit.edu/ alamo/pdf/2003-2002/2002/delAlamo\%20NL\%202002.pdf>. Acceso en: 16 ago. 2018.

ESCOBAR-PÉREZ, J.; CUERVO-MARTÍNEZ, A. Validez de contenido y juicio de expertos: una aproximación a su utilización. Avances en Medición, 2008. v. 6, p. 27-36. Disponible en:

$<$ http://www.humanas.unal.edu.co/psicometria/files/7113/8574/5708/Articulo3_Jui cio_de_expertos_27-36.pdf>. Acceso en: 14 jan. 2017.

GARCÍA, F. et al. Remote Laboratories for Electronics and New Steps in Learning Process Integration, 2016. En: INTERNATIONAL CONFERENCE ON REMOTE ENGINEERING AND VIRTUAL INSTRUMENTATION, $13^{\text {th }}, 2016$, Madrid, España.

GONZÁleZ, M. A.; PERDOMO, K. V.; PASCUAS, Y. Aplicación de las TIC en modelos educativos blended learning: una revisión sistemática de literatura. Revista Sophia, v. 13, n. 
1, p. 144-154, 2017. Disponible en: <http://revistas.ugca.edu.co/index.php/sophia/article/ view/364/1050> Acceso en: 23 mai. 2017.

HENRY, J. Laboratory Teaching via the World Wide Web. En Proceedings of the 1998 ASEE Southeastern Section Conference (180-187), 1998. Disponible en: <http://icee.usm. edu/icee/conferences/ASEE-SE-2010/Conference\%20Files/ASEE1998/Henry.pdf>. Acceso en: 20 ago. 2018.

HESSELINK, L. et al. CyberLab: Remote access to laboratories through the world-wideweb, 2000. Disponible en: <http://www.discoverlab.com/References/043.pdf>. Acceso en 19 ago. 2018.

LABSHARE. Benefits for students. 2017. Disponible en: <http://www.labshare.edu.au/remo telabs/students>. Acceso en: 13 set. 2017.

MARTÍN, G. Diseño e implementación de laboratorio remoto para la experimentación con el principio de Arquímedes mediante arquitectura asíncrona distribuida. 2013. Tesis (Grado en Ingeniería en Telecomunicaciones) - Universidad de Deusto, Bilbao, España.

SAN CRISTÓBAL, E. Metodología Estructura y Desarrollo de Interfaces Intermedias para la Conexión de Laboratorios Remotos y Virtuales a Plataformas Educativas. 2010 Disertación (Doctoral en Sistemas de Información) - Universidad Nacional de Educación a Distancia, España.

VELASQUEZ, Y.; RAMOS, O.; AMAYA, D. Technology used for the implementation of remote laboratories. International Journal of Applied Engineering Research, v. 11, n. 11, p. 7456-7461, 2016. Disponible en: <https://www.ripublication.com/ijaer16/ijaerv11 n11_41. pdf>. Acceso en: 07 mar. 2017.

WONG, H. et al. Mechatronics/process control remote laboratory, 2001. Disponible en: $<$ http://search.asee.org/search/fetch;jsessionid=vmv6ugjyqtd5?url=file\%3A\%2F\%2Flocalhost $\% 2 \mathrm{FE} \% 3 \mathrm{~A} \% 2 \mathrm{Fsearch} \% 2 \mathrm{Fconference} \% 2 \mathrm{~F} 25 \% 2 \mathrm{FAC} \% 25202001$ Paper672.PDF\&index=conf erence_papers\&space $=129746797203605791716676178 \&$ type $=$ application $\% 2 F p d f \&$ charset $>$ Acceso en: 16 ago. 2018.

ZAMORA, R. Laboratorios Remotos: Actualidad y tendencias futuras. Scientia et technica, v. 17, n. 51, p. 113-118, 2012. Disponible em: <http://www.redalyc.org/html/849/ 84923910017/>. Acceso en: 16 ago. 2016.

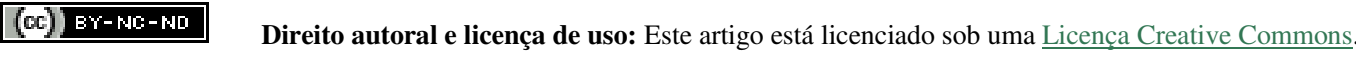

